\title{
Transverse Sectioning of Arabidopsis thaliana Leaves Using Resin Embedding
}

Shweta Kalve ${ }^{1,4 \#}$, Kumud Saini ${ }^{1 \#}$, Kris Vissenberg ${ }^{1}$, Tom Beeckman ${ }^{2,3}$ and Gerrit T. S. Beemster $^{1 *}$

${ }^{1}$ Department of Biology, University of Antwerp, Belgium; ${ }^{2}$ Department of Plant Systems Biology, VIB, Gent, Belgium; ${ }^{3}$ Department of Plant Biotechnology and Bioinformatics, Ghent University, Gent, Belgium; ${ }^{4}$ Present address: Department of Plant and Soil Sciences, Institute for Agricultural Biosciences, Oklahoma State University, Oklahoma, USA

*For correspondence: gerrit.beemster@uantwerpen.be

"These authors contributed equally to this work

[Abstract] The leaf is the major functional part of the shoot performing the bulk of photosynthetic activity. Its development is relatively plastic allowing the plant to adapt to environmental changes by modifying leaf size and anatomy. Moreover, a leaf is made up of various distinct cell layers, each having specialized functions. To understand functional adaptation and the development of the leaf it is essential to obtain cross sections throughout leaf development and at maturity (Kalve et al., 2014). Here, we describe a protocol for transverse sectioning of Arabidopsis thaliana leaves using resin embedding. This protocol provides a reliable platform to yield high quality images of cross sections allowing study of development of various tissue layers across the transversal axis of the leaf. As this method is an adaptation of the protocol developed for the Arabidopsis root tip by Beeckman and Viane (1999) and De Smet et al. (2004), it can easily be modified to accommodate other organs and species.

\section{Materials and Reagents}

1. Arabidopsis thaliana

2. Acetic Acid, Glacial (Certified ACS), Fisher Chemical (Fisher Scientific, catalog number: A38-212)

3. Formaldehyde solution (Sigma-Aldrich catalog, number: 47629)

4. Ethanol (VWR International, catalog number: 83813360)

5. Acetic anhydride (Sigma-Aldrich, catalog number: 320102)

6. TWEEN ${ }^{\circledR} 20$ (Sigma-Aldrich, catalog number: P9416)

7. $\mathrm{NaH}_{2} \mathrm{PO}_{4} \mathrm{H}_{2} \mathrm{O}$ (Fisher Scientific, catalog number: AC2717500190)

8. Technovit 7100 with hardener I and II (Heraeus Kulzer $\mathrm{GmbH}$, catalog number: 64709003)

9. Toluidine blue (EMD Millipore Corporation, catalog number: 1159300025)

10. DPX mounting medium (a mixture of Distyrene, a Plasticizer and Xylene) (SigmaAldrich, catalog number: M1289-10 ML) 
11. Object slides (superfrost plus adhesion slides) (Thermo Fisher Scientific, catalog number: 10143352)

12. Glass microtome knives (SPI Easy-cut Ultra/Glass, catalog number: 07668-BA)

13. Cover slips

14. Microcentrifuge tubes, PCR tubes or molds (Electron microscopy sciences, catalog number: 70900)

15. Phosphate buffer (see Recipes)

16. Technovit solution (see Recipes)

17. Technovit solution with hardener-II (see Recipes)

\section{Equipment}

1. Vacuum pump (Welch USA- BRS, catalog number: 2522C-02)

2. Vacuum jar

3. Tweezers

4. Heated plate (Bekso, model: TH3N)

5. Rotary microtome (Leica Reichert-Jung, catalog number: 2040)

6. Bright field microscope (ZEISS, model: Axio Scope A1) fitted with a digital camera (ZEISS, model: AxioCamCm1)

\section{Procedure}

1. Fixation: Dissect and fix whole Arabidopsis leaf blades under a vacuum in a solution of $5 \%$ acetic acid, $5 \%$ formaldehyde and $50 \%$ ethanol for an hour. Alternatively, ethanol:acetic anhydride (3:1) with a drop of Tween-20 can be used for fixation. Fixation is preferentially performed in glass vials to allow visualization of the leaves and multiple replicate leaves from a single stage/treatment can be treated in the same volume. The samples should sink as this is indicative of a good penetration of the fixative. Store the leaves overnight in the same fixation solution at $4{ }^{\circ} \mathrm{C}$.

2. Rinse: Rinse the fixed leaf samples with phosphate buffer ( $\mathrm{pH} 7.2)$.

3. Dehydration: Dehydrate the tissues by sequential incubations (Note 1) in a volume of ethanol sufficient to submerge freely specimens floating, without being constricted by the side walls of the container:

- $30 \%$ ethanol for $4 \mathrm{~h}$

- $50 \%$ ethanol for $4 \mathrm{~h}$

- $70 \%$ ethanol for $4 \mathrm{~h}$, overnight or sample can be stored at $4{ }^{\circ} \mathrm{C}$ for a maximum of 2 weeks

- $80 \%$ ethanol for $4 \mathrm{~h}$

- $90 \%$ ethanol for $4 \mathrm{~h}$

- $95 \%$ ethanol for $4 \mathrm{~h}$ or overnight at $4{ }^{\circ} \mathrm{C}$ 
- $98 \%$ ethanol for $1 \mathrm{~h}$

- $99 \%$ ethanol for $1 \mathrm{~h}$

- $100 \%$ ethanol for $1 \mathrm{~h}$. Repeat this step one more time.

4. Pre-infiltration: To replace the ethanol with Technovit 7100 resin (Figure 1a) perform following steps (Note 2):

- 30\% Technovit solution and $70 \%$ ethanol (consisting of $100 \%$ ethanol) for $15 \mathrm{~h}$

- 50\% Technovit solution and 50\% ethanol (consisting of 100\% ethanol) for $15 \mathrm{~h}$

- 70\% Technovit solution and $30 \%$ ethanol (consisting of $100 \%$ ethanol) for $15 \mathrm{~h}$

5. Infiltration: Replace the above solution with $100 \%$ Technovit solution and incubate for $15 \mathrm{~h}$ at room temperature.

6. Polymerization: Place the leaf samples in molds (Figure 1b-f) or microcentrifuge tubes (Figure 1g-h) and add Technovit solution with hardener-II. Let it polymerize overnight at $37^{\circ} \mathrm{C}$ in a closed environment i.e. cover the molds or cap microcentrifuge tubes to avoid evaporation (Note 3). Samples can be embedded horizontally in the molds as shown in Figure 1e or flat molds can be made between 2 overhead projection transparencies (Beeckman and Viane, 1999), which are then re-embedded vertically in microcentrifuge tubes.

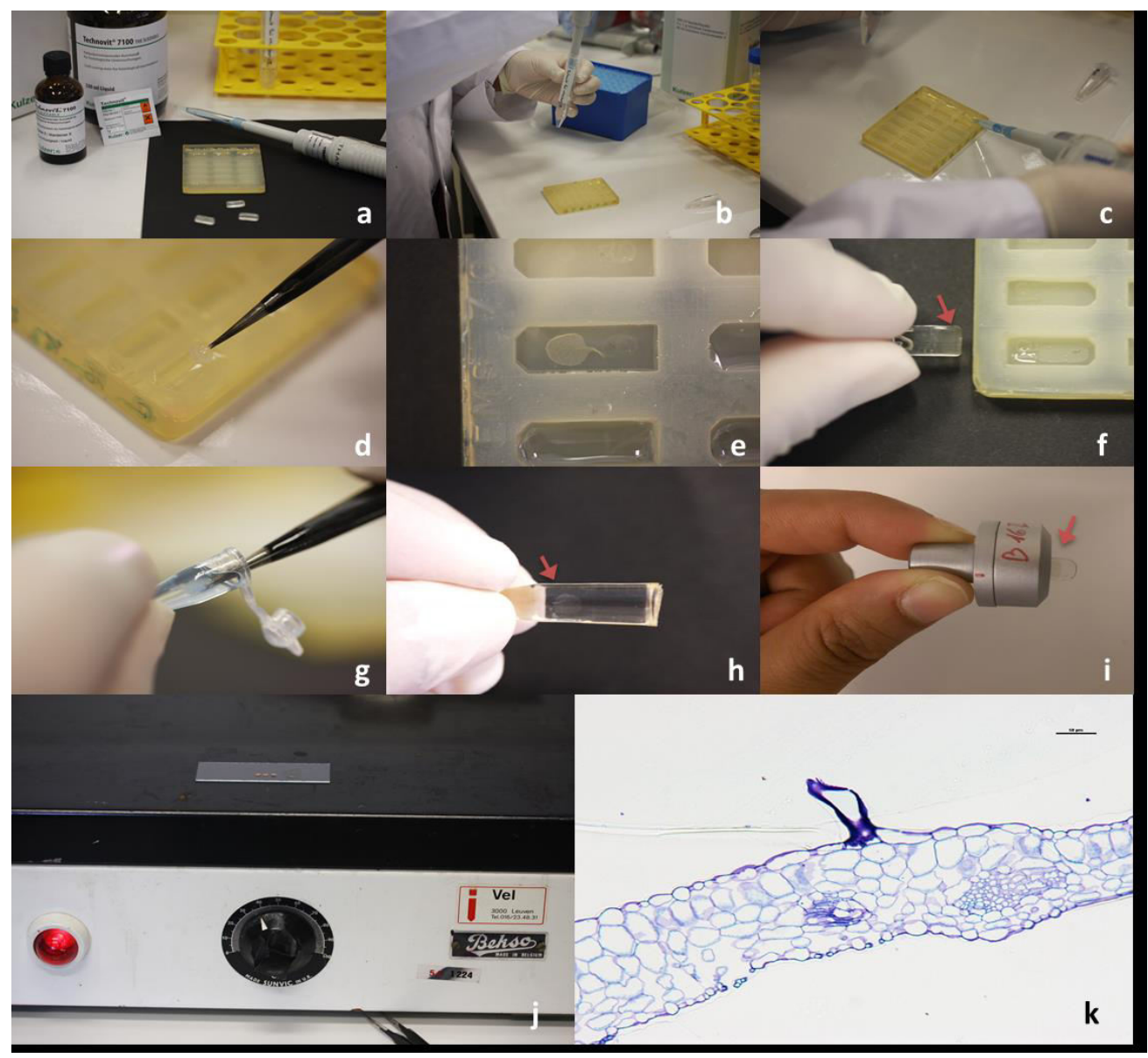

Figure 1. Subsequent steps of the protocol. a. overview of the work space; b-c. preparing and adding Technovit solution with hardener-II to the molds; d-e. placing the 
sample at the desired orientation; f. polymerized resin block containing sample (arrow); $\mathrm{g}$-h. alternatively PCR tubes can be used as molds; i. resin block attached to microtome sample holder; j. samples on a slide placed on the heated plate; k. transverse, stained section of leaf visualized using a bright field microscope.

7. Position the polymerized samples horizontally (Figure 1i) in the microtome to obtain the transverse sections. Make the sections of required thickness (in this case $2 \mu \mathrm{m}$ ) with a glass knife on the rotary microtome.

8. Take the piece of section with tweezers and place it into a drop of water on an object slide.

9. Place the object slide on the heated plate at $30-40^{\circ} \mathrm{C}$ and let it dry (Figure 1j).

10. Staining: Stain the sections on the object slide with a $0.05 \%(\mathrm{w} / \mathrm{v})$ solution of toluidine blue for $10 \mathrm{~min}$ and rinse with distilled water for $1 \mathrm{~min}$.

11. Dry the sections on the object slide at $30-40{ }^{\circ} \mathrm{C}$ on the heated plate and mount the cover slip using DEPEX as a mounting agent.

12. Take pictures of the sections at $20 x$ or $40 x$ magnification with a bright field microscope fitted with a digital camera (Figure $1 \mathrm{k}$ ).

\section{$\underline{\text { Notes }}$}

1. All the sequential incubation steps should be performed without changing the container and removing the solutions between incubation steps by pipette to avoid tissue damage.

2. Samples can be pre-stained by neutral red or toluidine blue just before embedding to aid in visualization, for desired orientation.

3. Technovit resin embedding is compatible with a broad range of stainings including immunolocalisation, enzyme histochemistry, in situ hybridization, and a range of classical histological stains (Willbold and Witte, 2010).

\section{Recipes}

1. Phosphate buffer

Add $13.799 \mathrm{~g} \mathrm{NaH}_{2} \mathrm{PO}_{4} \cdot \mathrm{H}_{2} \mathrm{O}$ in $1 \mathrm{~L}$ water

Adjust the $\mathrm{pH}$ to 7.2

2. Technovit solution

Add $1 \mathrm{~g}$ hardener-I in $100 \mathrm{ml}$ Technovit 7100, protect it from light

It can be stored at $4{ }^{\circ} \mathrm{C}$ for few weeks

3. Technovit solution with hardener-II

Add $1 \mathrm{ml}$ hardener-II in $15 \mathrm{ml}$ Technovit solution, mix it by stirring and keep on ice to avoid polymerization 
Make the fresh solution and use it immediately

\section{Acknowledgements}

We would like to thank Maria Njo for technical support. This work was supported by a concerted research activity (GOA) research grant, 'A Systems Biology Approach of Leaf Morphogenesis' from the research council of the University of Antwerp and an Interuniversity Attraction Poles project (IAP7/29, MARS) from the Belgian Federal Science Policy Office (BELSPO). Shweta Kalve was funded by a training grant from the Department of Science and Education of the Flemish Government. The authors acknowledge the financial support of the Research Foundation-Flanders (FWO-Vlaanderen).

\section{$\underline{\text { References }}$}

1. Beeckman, T. and Viane, R. (2000). Embedding thin plant specimens for oriented sectioning. Biotech Histochem 75(1): 23-26.

2. De Smet, I., Chaerle, P., Vanneste, S., De Rycke, R., Inze, D. and Beeckman, T. (2004). An easy and versatile embedding method for transverse sections. J Microsc 213 (Pt 1): 76-80.

3. Kalve, S., Fotschki, J., Beeckman, T., Vissenberg, K. and Beemster, G. T. (2014). Three-dimensional patterns of cell division and expansion throughout the development of Arabidopsis thaliana leaves. J Exp Bot 65(22): 6385-6397.

4. Willbold, E. and Witte, F. (2010). Histology and research at the hard tissue-implant interface using Technovit 9100 New embedding technique. Acta Biomater 6(11): 4447-4455. 